\title{
Identifying antibiotic stewardship interventions to meet the NHS England CQUIN: an evaluation of antibiotic prescribing against published evidence-based antibiotic

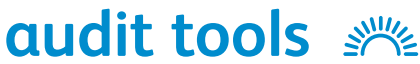

\author{
Authors: Neil Powell, ${ }^{A}$ Kate McGraw-Allen, ${ }^{B}$ Alasdair Menzies, ${ }^{C}$ Bradley Peet, ${ }^{D}$ Callie Simmonds ${ }^{E}$ \\ and Abigail Wild ${ }^{\mathrm{F}}$
}

Evidence-based audit tools were used to identify the antibiotic stewardship improvements necessary to meet the NHS England targets in a 750-bed teaching hospital.

Antibiotic prescribing was reviewed against published evidencebased audit tools for 139 patients treated with antibiotics. Severe community-acquired pneumonia (CAP) median course length was 8.5 days. Ninety-six percent of non-severe CAP patients were initiated on intravenous antibiotics (IV); median antibiotic course length 9 days. Twenty-six percent of urinary tract infection (UTI) patients without an indwelling catheter met the UTI diagnostic criteria. IV antibiotics initiated in $79 \%$ patients with other infections. Of these, $17 \%$ met the IV to oral switch criteria at 72 hours but were not switched. On average, antibiotic courses were $19 \%$ longer than recommended. Three key areas for improvement consist of: (a) implement the National Institute of Health and Care Excellence UTI Quality Standard - only $38 \%$ of patients treated for UTI met the UTI definition; (b) ensure antibiotic course lengths are in line with local prescribing guidelines - antibiotics were continued for $14 \%$ longer than recommended in local guidelines; (c) switch antibiotic therapy to oral when switch criteria met - $17 \%$ percent of patients initiated on IV antibiotics were eligible for oral switch by 72 hours and were not switched.

KEYWORDS: Antibiotic stewardship, audit

Authors: ${ }^{\text {A }}$ consultant antimicrobial pharmacist, Royal Cornwall Hospitals NHS Trust, Truro, UK and National Institute for Health Research Health Protection Research Unit (NIHR HPRU) in healthcare associated infections and antimicrobial resistance at Imperial College London in partnership with Public Health England (PHE), London, UK; ${ }^{\text {B }}$ foundation year one doctor, Royal Cornwall

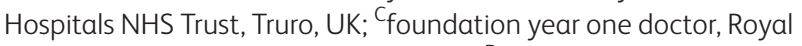
Cornwall Hospitals NHS Trust, Truro, UK; ${ }^{\text {foundation year two }}$ doctor, Royal Cornwall Hospitals NHS Trust, Truro, UK; Efoundation year one doctor, Royal Cornwall Hospitals NHS Trust, Truro, UK;

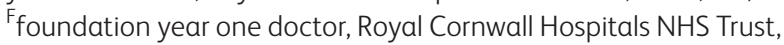
Truro, UK

\section{Introduction}

Antimicrobial resistance (AMR), with an estimated 10 million attributable deaths by $2050^{1}$ is a global concern the United Nations have pledged to tackle. ${ }^{2}$ Antimicrobial use is the single biggest driver for AMR with broad-spectrum agents and protracted antibiotic courses exerting the greatest resistance pressure. ${ }^{3-5}$ Between $20-50 \%$ of inpatient antibiotic prescribing is inappropriate. ${ }^{6,7}$ The US National Strategy for Combating Antibiotic-resistant Bacteria calls for reductions in inappropriate prescribing by $50 \%$ by $2020,{ }^{8}$ and this is also an objective for the UK. ${ }^{9}$ Unnecessary antibiotic prescribing unnecessarily increases patients' risk of Clostridium difficile-associated diarrhoea ${ }^{10}$ and side effects. ${ }^{11}$ The NHS has responded through its Commissioning for Quality and Innovation (CQUIN) scheme. The 2017 CQUIN sets a goal for trusts to reduce clinically inappropriate antibiotic consumption with targets set to reduce the use of piperacillin/ tazobactam, carbapenem and total antibiotic use by $1 \%$ compared to 2016. A portion of healthcare provider income is conditional on meeting the CQUIN targets.

Definitions of inappropriate antibiotic prescribing are subjective ${ }^{12}$ and a standard set of definitions is needed. One group has developed evidenced-based audit tools enabling organisations to review appropriateness of antibiotic prescribing against a peer-reviewed standard. ${ }^{13}$

This study sets out to review antibiotic prescribing in an English hospital against published evidence-based antibiotic stewardship audit tools. ${ }^{13}$ Recommended changes to practice will be made to the Hospital Management Board with an emphasis on changes that will enable the hospital to meet the financially incentivised CQUIN antibiotic consumption reduction targets. ${ }^{14}$

Methods

\section{Setting}

This evaluation of antibiotic prescribing was conducted at a 750bed teaching district general hospital in England. The hospital has a comprehensive antimicrobial stewardship programme which includes: implementation of the national stewardship guideline, ${ }^{15}$ a restricted antimicrobial system requiring medical microbiologist authorisation for use of restricted antibiotics for non-pre- 
approved indications; ${ }^{16}$ daily antibiotic pharmacist antimicrobial stewardship ward rounds; daily ward pharmacist ward rounds in which antibiotic stewardship is one of their duties and periodic medical microbiologist ward rounds. The hospital has an electronic prescribing and medication administration (EPMA; JAC Computer Services Ltd) system deployed in all inpatient areas (except critical care) with non-mandated antibiotic indication field and nonmandated antibiotic stop date or review date field. Compliance with the trust requirement to document antibiotic indication and stop or review dates on the prescribing system is regularly audited and maintained at approximately $85 \%$ and $83 \%$ respectively.

\section{Data extraction}

All adult and paediatric patients discharged (including deaths) from the study hospital in February 2017 who had received at least 24 hours antibiotics as an inpatient were identified via the EPMA system, identifying 964 patients. Every 10 th patient plus all prescriptions from the remaining patients with a communityacquired pneumonia (CAP) or urinary tract infection (UTI) indication were included which identified 139 cases. Paper medical records for all identified patients were requested.

\section{Review of antibiotic prescribing}

The antibiotic management was reviewed against three of four audit tools. ${ }^{13}$ 'Assessment of appropriateness of antibiotic use for resistant Gram-positive infections' tool was excluded due to the low prevalence of vancomycin-resistant enterococcus and methicillin-resistant Staphylococcus aureus in the study hospital.

We made the following amendments to the review tools. ${ }^{13}$ To the CAP tool we included a CURB 65 score $\geq 3$ to reflect UK practice. ${ }^{17}$ CURB 65 estimates CAP mortality, patients scoring one point for each of: confusion, urea $>7 \mathrm{mmol} / \mathrm{L}$, respiratory rate $>30 \mathrm{bpm}$, systolic blood pressure below $100 \mathrm{mmHg}$ and age over 65 years. Patients with a CURB 65 score of 3 or above or admitted to the intensive care unit met the study criteria for severe CAP.

To the 'Assessment of appropriateness of antibiotics for other inpatient antibiotics' tool we added a final question 'Total duration of antibiotic including discharge prescription'.

To the assessment 'Assessment of appropriateness of antibiotics for urinary tract infections (UTI)' tool we added total antibiotic course to the inpatient course question.

We removed the following question from the CAP and 'other inpatient antibiotics' tool: 'Was the patient initially prescribed an intravenous (IV) antibiotic with good oral bioavailability?' This was because IV antibiotics are recommended at the study hospital according to severity of infection not oral bioavailability. The IV to oral switch criteria were amended to represent that of the study hospital (Supplementary file 1).

\section{Results}

One hundred and thirty five patients' medical records from 16 different specialties were reviewed with a mean age of 65 years (range: 2 months - 101 years).

\section{Community-acquired pneumonia}

\section{Severe CAP}

Seven of 10 patients ( $70 \%$ ) with severe CAP were commenced on antibiotics consistent with the study hospital's antibiotic
Table 1. Local antibiotic prescribing guidelines for

community-acquired pneumonia (CAP) and urinary

tract infections (UTI)

$\begin{array}{ll}\begin{array}{l}\text { Infection } \\ \text { Severe community-acquired } \\ \text { pneumonia (CAP) }\end{array} & \begin{array}{l}\text { Antibiotic regimen } \\ \text { Piperacillin/tazobactam + } \\ \text { doxycycline/clarithromycin } \\ \text { Penicillin allergy } \\ \text { Levofloxacin }\end{array} \\ \text { Non-severe CAP } & \begin{array}{l}\text { Amoxicillin +/-doxycyline / } \\ \text { clarithromycin } \\ \text { Penicillin allergy } \\ \text { Clarithromycin or doxycyline }\end{array} \\ \text { Lower urinary tract infection (UTI) } & \text { Nitrofurantoin or trimethoprim } \\ \text { Upper UTI } & \text { Amoxicillin + gentamicin }\end{array}$

prescribing guidelines (Table 1), all initiated on IV antibiotics. Non-compliant regimens were single agent regimens containing one of piperacillin/tazobactam, ceftriaxone or amoxicillin. The median antibiotic course length was 8.5 days, ranging from 3 to 31 days. One adult patient received 11 days, one paediatric patient received 31 days for a pneumonia complicated by empyema and the remaining patients received 10 or fewer days of therapy. Three patients (30\%) had sputum cultures sent and nine $(90 \%)$ had blood cultures sent prior to administration of antibiotics; one patient (10\%) had a urine legionella and pneumococcal antigens ordered.

Two (20\%) severe CAP patients were switched from IV to oral antibiotics by 72 hours; the remaining patients had clinical indicators for remaining on IV antibiotics in accordance with the study hospital's IV to oral switch guidelines.

An infecting organism was identified in the blood cultures of three $(30 \%)$ patients, all sensitive to the prescribed antibiotic. None of the patients were switched to available narrower spectrum agents at the time of sensitivity result.

\section{Non-severe CAP}

Twenty-three patients had non-severe CAP. ${ }^{17}$ Nine (39\%) patients' initial antibiotic regimens were in accordance with the trust's antibiotic guidelines (Table 1). One patient was prescribed meropenem on medical microbiologist advice and so deemed to be within trust recommended guidelines. All fourteen (61\%) noncompliant regimens contained piperacillin/tazobactam instead of the recommended amoxicillin. Twenty-two (96\%) were initiated on IV antibiotics, with seven (32\%) patients switched to oral antibiotics within 72 hours. Two patients (13\%) remained on IV antibiotics beyond 72 hours without an appropriate clinical reason.

The median antibiotic course length was 9 days, ranging from 3 to 24 days. Of these, nine patients received 7 days or less, nine patients received 8 to 10 days therapy and five patients received between 11 days and 24 days. The reasons for these extended course lengths were not captured by the audit.

\section{UTI}

Twenty-nine patients (mean age 83 years, range 68 to 93 years) were treated for a UTI across 10 specialties. Eighteen patients 
were female and 11 were male. Seven patients had a urinary catheter in situ.

Of the patients without a urinary catheter, six ( $27 \%$ ) patients had at least two signs or symptoms of UTI; five patients met upper UTI diagnostic criteria and one patient met lower UTI diagnostic criteria. $^{18}$

Of the seven patients with a urinary catheter, five (71\%) had at least one sign or symptom consistent with a catheter-associated UTI (CAUTI).

Antibiotic course lengths for the 11 patients meeting the diagnostic criteria for UTI were as follows: five patients with upper UTI median 4 days (range 3-15); one female patient with lower UTI 3 days; five patients with CAUTI median 5 days (range 3-10 days). None were prescribed piperacillin/tazobactam or meropenem.

\section{Other infections}

Seventy-three patients did not have a CAP or UTI diagnosis: 43 $(58.9 \%)$ female and $30(41.1 \%)$ male, mean age 65 , ranging from 2 months to 94 years, treated in 17 specialties within the trust. Indications for the antibiotics were documented in the medical notes for 71 (97.2\%) patients (Table 2). Two patients did not have an indication documented in the medical notes and were excluded.

Fifty-three (75\%) antibiotic regimens were in line with trust guidelines. Of the 20 (25\%) non-compliant regimens two contained piperacillin/tazobactam instead of a narrower spectrum agent recommended in trust guidelines. One patient was prescribed meropenem on medical microbiology advice. Fortynine patients $(69 \%)$ had cultures taken, but of these 14 (29\%) had antibiotics administered before the collection of cultures.

Fifty-six of $71(79 \%)$ patients were initiated on IV antibiotics and $15(21 \%)$ on oral antibiotics. Twenty-six of $56(46 \%)$ patients initiated on IV antibiotics were switched to oral by 72 hours. Of the 30 of $56(54 \%)$ patients that were not switched to oral by 72 hours, five (17\%) patients had met local IV to oral switch criteria. The other 25 of 30 (83\%) patients not switched to oral at 72 hours met at least one IV to oral switch instability parameter (Supplementary file 1).

Median inpatient antibiotic duration was 6 days. Median total antibiotic duration, ie including discharge antibiotics, was 7 days (excluding one patient on lifelong antibiotics). Two hundred and twenty-seven days of antibiotic therapy were prescribed against the study hospital's local course length recommendation of 190 days ( $19 \%$ excess days) (Table 3 ). Across the three audit areas, CAP, UTI and 'other infections', 596 days of therapy were prescribed against an expected 524 (14\% excess days) (Table 3 ).

\section{Discussion}

\section{Severe CAP}

All patients with severe CAP were started on IV therapy, in line with national recommendations. ${ }^{17}$

The national guidelines ${ }^{17}$ recommend blood and sputum cultures in all patients with severe CAP. While $90 \%$ had blood cultures sent, only $30 \%$ had sputum sent, an area for local practice improvement. The usefulness of sputum cultures relies on good quality samples which are not always obtained. If more sputum cultures were obtained and sent for culture, and of good quality, it would aid identification of S pneumoniae and Haemophilus influenzae, two common respiratory pathogens. ${ }^{19,20}$ Timely culture of sputum and antimicrobial sensitivities will enable targeted antimicrobial therapy thereby optimising patient care and reducing unnecessary prescribing. Only one (10\%) severe CAP patient had urine legionella and pneumococcal antigens ordered as recommended in national guidelines ${ }^{17}$ and all three opportunities to narrow the antibiotic spectrum were missed; ordering sputum cultures and narrowing antibiotic spectrum according to microbiology reports are both areas for local practice improvement.

The national guidance recommends an IV to oral switch as soon as clinical improvement has occurred and apyrexial for 24 hours. ${ }^{17}$ Despite only two $(20 \%)$ patients being switched to oral antibiotics by 72 hours, there were appropriate clinical indicators for the other eight patients, according to local trust IV to oral switch guidelines, to remain on IV therapy.

The median antibiotic course length for patients with severe CAP was 8.5 days against national recommended $7-10$ day course length. Therefore, the average severe CAP course lengths in this study were in accordance with recommended course lengths.

\section{Non-severe CAP}

Of those patients treated for non-severe CAP (23 in total), 39\% were initiated on antibiotics compliant with trust guidelines. All non-compliant regimens contained piperacillin/tazobactam. It is possible that this over prescribing of broad-spectrum antibiotics is driven by the practice of administering broad spectrum antibiotics to patients with clinical signs of systemic inflammatory response syndrome (SIRS). The administration of these broad spectrum antibiotics prior to a definitive diagnosis, in line with national ${ }^{14,21}$ and local trust guidance for managing sepsis, presents an antibiotic stewardship challenge. The British Thoracic Society (BTS) CAP guidelines recommend the use of oral antibiotics for patients admitted to hospital with non-severe CAP. ${ }^{17}$ The reasons for the overuse of intravenous antibiotics by doctors has been identified to be: risk of litigation or complaints if patient expectations were not met, hierarchy of the medical team structure providing limited opportunities for de-escalation of antibiotics and the perception that IV antibiotics were more potent. ${ }^{22}$ Broom et al concluded the IV to oral switch interventions should include strategies to demystify IV versus oral antibiotic efficacy, engage consumers (patients) around the negative effects of IV antibiotic overuse and examine strategies to streamline team decision-making. ${ }^{22}$ If we can rationalise the overuse of broad-spectrum IV antibiotics, in this case piperacillin/tazobactam, it will enable the trust to meet the CQUIN target and reduce resistance to these vital medications. $^{23}$

The authors recognise the challenges of countering the drive to prescribe broad-spectrum IV antibiotics in those with SIRS; however, the diagnosis of non-severe CAP should prompt an IV antibiotic review and initiate an oral switch. Thirty-two percent of the patients with non-severe CAP initiated on IV antibiotics were switched to oral antibiotics within 72 hours. There is an opportunity to switch antibiotics on the consultant post take ward round to a narrow spectrum, oral antibiotic in those with a definitive diagnosis of non-severe CAP.

The BTS Guidelines ${ }^{17}$ recommended a 7-day antibiotic course for uncomplicated pneumonia. More recent NICE guidelines ${ }^{17}$ recommend 5 days of antibiotics for low severity CAP, and a 5-7 day course for moderate to high severity CAP. Non-severe CAP combines both low and moderate severity as categorised by the NICE guidelines. Therefore, antibiotic course lengths for 
Table 2. Infections requiring antibiotics, including type, course length, and percentage of excess treatment

\begin{tabular}{|c|c|c|c|c|c|c|}
\hline System infected & Infection & $\begin{array}{l}\text { Number of } \\
\text { patients }\end{array}$ & $\begin{array}{l}\text { Median } \\
\text { inpatient } \\
\text { antibiotic } \\
\text { duration } \\
\text { days (range } \\
\text { days) }\end{array}$ & $\begin{array}{l}\text { Median total } \\
\text { antibiotic } \\
\text { course days } \\
\text { (course } \\
\text { length range } \\
\text { in days) }\end{array}$ & $\begin{array}{l}\text { Trust } \\
\text { recommended } \\
\text { duration (days) }\end{array}$ & $\begin{array}{l}\text { Median days of } \\
\text { therapy received } \\
\text { for infection / } \\
\text { recommended } \\
\text { (percentage excess) }\end{array}$ \\
\hline \multirow{4}{*}{$\begin{array}{l}\text { Skin and soft } \\
\text { tissue }\end{array}$} & Dog bite & 1 & $3(\mathrm{NA})$ & $10(10)$ & 7 & $10 / 7(43 \%)$ \\
\hline & Facial cellulitis & 2 & $4.5(2-7)$ & $7(7)$ & 10 & $7 / 10(-30 \%)$ \\
\hline & Orbital cellulitis & 1 & $5(n / a)$ & $12(n / a)$ & 10 & $12 / 10(20 \%)$ \\
\hline & Cellulitis & 1 & $15(n / a)$ & $15(n / a)$ & $7-10$ & $15 / 10(50 \%)$ \\
\hline Sepsis & Source unknown adult & 7 & $6(4-11)$ & $7(5-11)$ & 7 & $7 / 7(0 \%)$ \\
\hline Obstetrics & Intrapartum pyrexia & 2 & $2.5(2-3)$ & $7.5(7-8)$ & 7 & 7.5/7 (7\%) \\
\hline \multirow[t]{4}{*}{ Respiratory } & HAP & 21 & $7(2-35)$ & $8(6-35)$ & 7 & $8 / 7(14 \%)$ \\
\hline & IECOPD & 3 & $5(2-9)$ & $7(5-9)$ & 5 & $7 / 5(40 \%)$ \\
\hline & Bronchitis & 8 & $5.5(2-10)$ & $7(2-17)$ & 7 & $7 / 7(0 \%)$ \\
\hline & Aspiration pneumonia & 1 & $7(n / a)$ & $7(n / a)$ & 7 & $7 / 7(0 \%)$ \\
\hline \multirow[t]{5}{*}{ Gastrointestinal } & Diverticulitis & 4 & $4(1-4)$ & $6(5-8)$ & 7 & $6 / 7(-14 \%)$ \\
\hline & $\begin{array}{l}\text { Intra-abdominal } \\
\text { infection }\end{array}$ & 2 & $5.5(5-6)$ & $9(5-13)$ & 7 & $9 / 7(28 \%)$ \\
\hline & $\mathrm{GDH}+$ & 1 & $5(n / a)$ & $5(n / a)$ & $\mathrm{n} / \mathrm{a}$ & $\mathrm{n} / \mathrm{a}$ \\
\hline & Cholangitis & 3 & $4(4-6)$ & $6(4-11)$ & 7 & $6 / 7(-14 \%)$ \\
\hline & Cholecystitis & 3 & $4(3-7)$ & $6(5-15)$ & 7 & $6 / 7(-14 \%)$ \\
\hline \multirow[t]{2}{*}{ ENT } & Mastoiditis & 1 & $3(n / a)$ & $13(n / a)$ & $7-14$ & Within range \\
\hline & Acute otitis media & 1 & $2(n / a)$ & $12(n / a)$ & 7 & $12 / 7(71 \%)$ \\
\hline \multirow[t]{14}{*}{ Others } & $\begin{array}{l}\text { Joint replacement, } \\
\text { prophylaxis }\end{array}$ & 1 & $6(n / a)$ & $6(n / a)$ & 1 & $6 / 1(600 \%)$ \\
\hline & $\begin{array}{l}\text { Suspected neonatal } \\
\text { sepsis }\end{array}$ & 1 & $3(n / a)$ & $3(n / a)$ & $\begin{array}{l}48 \text { hours then } \\
\text { review }\end{array}$ & $\mathrm{n} / \mathrm{a}$ \\
\hline & Diabetic foot & 1 & $7(n / a)$ & $\mathrm{n} / \mathrm{a}$ & Up to 3 months & Within range \\
\hline & Multiple lung abscesses & 1 & $2(n / a)$ & $13(n / a)$ & $\begin{array}{l}14 \text { Seek micro } \\
\text { advice }\end{array}$ & $\mathrm{n} / \mathrm{a}$ \\
\hline & $\begin{array}{l}\text { Surgical wound } \\
\text { infection }\end{array}$ & 1 & $11(n / a)$ & $11(n / a)$ & $\begin{array}{l}48 \text { hours then } \\
\text { review }\end{array}$ & $\mathrm{n} / \mathrm{a}$ \\
\hline & Tooth infection & 1 & $9(n / a)$ & $9(n / a)$ & 7 & $9 / 7(28 \%)$ \\
\hline & $\begin{array}{l}\text { Cystic fibrosis } \\
\text { exacerbation }\end{array}$ & 1 & $27(n / a)$ & $27(n / a)$ & 14 & $27 / 14(93 \%)$ \\
\hline & Orchitis & 1 & $5(n / a)$ & $12(n / a)$ & 14 & $12 / 14(-14 \%)$ \\
\hline & Tubo-ovarian abscess & 1 & 3 & $5(n / a)$ & 7 & $5 / 7(-29 \%)$ \\
\hline & Severe CAP & 10 & - & $8.5(3-31)$ & $7-10$ & $8.5 / 10(-15 \%)$ \\
\hline & Non-severe CAP & 23 & - & $9(3-24)$ & $5-7$ & $9 / 7(29 \%)$ \\
\hline & Lower UTI & 1 & - & $3(n / a)$ & 3 & $\mathrm{n} / \mathrm{a}$ \\
\hline & Upper UTI & 5 & - & $4(3-15)$ & $7-10$ & $4 / 10(-60 \%)$ \\
\hline & CAUTI & 5 & - & $5(3-10)$ & 7 & $5 / 7(-29 \%)$ \\
\hline
\end{tabular}

$\mathrm{CAP}=$ community-acquired pneumonia; $\mathrm{CAUTI}=$ catheter-associated urinary tract infection; $\mathrm{CF}$ = cystic fibrosis; $\mathrm{GDH}=$ glutamic acid dehydrogenase (C difficile); $\mathrm{HAP}=$ hospital-acquired pneumonia; IECOPD = infective exacerbation of chronic obstructive disease; UTI = urinary tract infection, NA = not available 
Table 3. Days of antibiotic therapy prescribed

against expected days of antibiotic therapy as per

local antibiotic prescribing guidelines

$\begin{array}{llll}\text { Infection group } & \begin{array}{l}\text { Days of } \\ \text { antibiotic } \\ \text { therapy } \\ \text { prescribed }\end{array} & \begin{array}{l}\text { Expected days } \\ \text { according } \\ \text { to local } \\ \text { guidelines }\end{array} & \begin{array}{l}\text { Excess days } \\ \text { (percentage) }\end{array} \\ \text { Other infections } & 227 & 190 & 37(19 \%) \\ \text { All CAP } & 271 & 261 & 10(4 \%) \\ \text { All UTI } & 98 & 73 & 25(34 \%) \\ \text { Total } & 596 & 524 & 72(14 \%)\end{array}$

non-severe CAP were protracted. Local promotion of national CAP guidelines, educating medical, pharmacy and nursing staff of the recommended antibiotic course lengths may optimise practice, potentially reducing unnecessary prescribing.

\section{UTI}

UTI is a clinical diagnosis made on presenting symptoms (eg dysuria, frequency, suprapubic tenderness). ${ }^{18}$ Patients over 65 years old, without an indwelling catheter, meet the UTI diagnosis criteria if two or more signs or symptoms consistent with a UTI are present. ${ }^{18}$ Only 6 of $22(27 \%)$ of non-catheterised patients treated for a UTI had at least two signs or symptoms of UTI, and were therefore consistent with a UTI diagnosis. This may be due to poor documentation of signs and symptoms in the medical notes or due to an absence of UTI signs and symptoms, suggesting over diagnosis and overtreatment of UTIs in this elderly study population, a problem reported elsewhere. ${ }^{18}$ Caterino et al reported that only $24 \%$ of $65-84$-year-old patients and $17 \%$ of $>85$-year-olds presenting to the emergency department in the USA given a UTI diagnosis, had urinary symptoms. ${ }^{24}$ Another study reports only $23 \%$ patients across US acute care hospitals with a UTI diagnosis had documented symptoms of UTI ${ }^{25}$ A UK quality improvement study increased the percentage of UTI patients meeting the SIGN UTI guideline criteria from $31.3 \%$ to $42.1 \%{ }^{26}$ Improving the diagnosis of UTI presents an opportunity to improve antibiotic stewardship.

Five of seven $(71 \%)$ patients with an indwelling urinary catheter had at least one sign or symptom consistent with a CAUTI diagnosis. ${ }^{18}$ There is little evidence to support current criteria for diagnosing UTI in catheterised patients. ${ }^{18}$ The SIGN clinical algorithm for suspected UTI in catheterised residents in nursing homes suggests that the presence of one of the following symptoms should prompt consideration of antibiotic therapy: rigors, new costovertebral tenderness or new onset delirium. ${ }^{18}$

The study hospital antibiotic course length guidance varies dependent on gender and site of UTI. Recommended course length for females patients with a lower UTI is 3 days, males is 7 days and all patients with an upper UTI is 7-10 days, CAUTI patients is 7 days. ${ }^{18}$ Median course lengths in this study were shorter than recommended in trust guidelines but the number of patients meeting diagnostic criteria were small.

\section{Others}

Overall, including UTI and CAP, 596 days of therapy were prescribed against an expected 524 days ( $14 \%$ excess days).
Reducing antibiotic courses lengths in line with recommendations would reduce antibiotic resistance pressure, side effects and hospital length of stay, thereby improving patient care. ${ }^{27}$

For patients with hospital-acquired pneumonia (HAP), the median duration of antibiotic therapy was 7 days as an inpatient and 8 days in total. Local antibiotic guidelines recommend a total course length of 7 days for $\mathrm{HAP}^{28,29}$ For patients with an infective exacerbation of COPD (IECOPD), the median antibiotic duration was 5 days as an inpatient and 7 days in total. Local antibiotic prescribing guidelines recommend 5 days, with international guidance $^{30}$ recommending 5-7 days. On average, patients with HAP and IECOPD had completed their antibiotic course prior to discharge, then supplied with further, unnecessary therapy on discharge. These extended course lengths may be due to a failure to count how many days patients had received as an inpatient, or prescription for discharge 'just in case'. Discharge prescriptions present doctors with an opportunity to review antibiotic course lengths and pharmacy staff to question protracted antibiotic course lengths.

Patients with diverticulitis were prescribed a median course length of 4 days as in inpatient and 6 days in total. The recommended course length in the local trust guidelines is 7 days. Early cessation of antibiotic therapy if infection was ruled out may be the reason for the low median course length.

Forty-nine of 71 (69\%) patients had cultures taken of which 49 patients (29\%) had received antibiotics prior to obtaining the culture samples. Administering antibiotics prior to the taking of blood cultures increases the likelihood of false-negative blood culture results, ${ }^{31}$ presenting an obvious risk to patient care.

A large proportion (79\%) of patients were commenced on IV antibiotics. Forty-six percent of these patients were switched to oral antibiotics by 72 hours. Seventeen percent of the patients who were eligible to switch to oral antibiotics at 72 hours, according to local IV to oral switch guidelines, were not switched. This is lower than the $34 \%$ of patients on IV antibiotics deemed eligible for oral switch in a UK study of six trusts where the review was done on antibiotic stewardship ward rounds using similar IV to oral switch criteria to those we used although the timing was not specified to be at 72 hours. ${ }^{32}$ These missed opportunities to switch from IV to oral may be because the antibiotics were not reviewed, a risk when staffing is low eg weekends, or due to inexperienced junior doctors not confident to use oral agents.

The study was conducted in order to identify key areas of focus in order to meet the CQUIN targets with the limited antibiotic stewardship resource available. This study is limited by the small number of patients reviewed making the interpretation of the individual infections within the 'other' cohort difficult, but nonetheless key areas for improvement have emerged enabling the antibiotic stewardship team to focus their efforts with a view to re-audit and evaluate intervention success after 12 months.

\section{Conclusion}

The use of audit tools such as those developed by Spivak et al ${ }^{13}$ have enabled us to identify stewardship practices amenable to change that would likely impact on patient care and the wider antibiotic resistance agenda.

We identified four key areas for improvement; implement the NICE UTI Quality Standard ${ }^{33}$ - only $38 \%$ of patients treated for UTI met the UTI definition; ensure antibiotic course lengths are in line with local prescribing guidelines - antibiotics were continued for 
$14 \%$ longer than recommended in local guidelines; switch antibiotic therapy to oral when switch criteria met $-8 \%$ of all patients who remained on IV antibiotics at 72 hours were eligible for oral switch and were not switched; switch antibiotics to narrowest spectrum agent once sensitivities reported. Work streams to address these areas for improvement will be set up and the audit repeated in 12 months.

\section{Supplementary material}

Additional supplementary material may be found in the online version of this article at www.clinmed.rcpjournal.org:

S1 - IV to oral switch criteria of the study hospital

\section{References}

1 O'Neill J. Tackling drug-resistant infections globally: Final report and recommendations. Review on Antimicrobial Resistance, 2016.

2 Nations United. Draft political declaration of the high-level meeting of the general assembly on antimicrobial resistance. UN, 2016

3 Chastre J, Wolff M, Fagon JY et al. Comparison of 8 vs 15 days of antibiotic therapy for ventilator-associated pneumonia in adults: a randomized trial. JAMA 2003;290:2588-98.

4 Singh N, Rogers P, Atwood CW, Wagener MM, Yu VL. Short-course empiric antibiotic therapy for patients with pulmonary infiltrates in the intensive care unit: a proposed solution for indiscriminate antibiotic prescription. Am J Respir Crit Care Med 2000;162:505-11.

5 Public Health England. English surveillance programme for antimicrobial utilisation and resistance (ESPAUR) Report 2016. PHE, 2016. www.gov.uk/government/publications/english-surveillanceprogramme-antimicrobial-utilisation-and-resistance-espaur-report [Accessed 26 October 2017].

6 Davey P, Brown E, Fenelon L et al. Interventions to improve antibiotic prescribing practices for hospital inpatients. Cochrane Database Syst Rev 2005;19:CD003543.

7 Willemsen I, Groenhuijzen A, Bogaers D et al. Appropriateness of antimicrobial therapy measured by repeated prevalence surveys. Antimicrob Agents Chemother 2007;51:864-7.

8 The White House. National Strategy for Combating Antibiotic Resistant Bacteria. The White House, 2014.

9 Prime Minister's Office. G7 2016 in Japan: PM press statement. www.gov.uk/government/speeches/g7-2016-in-japan-pm-pressstatement [Accessed 26 October 2017].

10 Pereira JB, Farragher TM, Tully MP et al. Association between Clostridium difficile infection and antimicrobial usage in a large group of English hospitals. Br J Clin Pharmacol 2014;77:896-903.

11 Tamma PD, Avdic E, Li DX, Dzintars K, Cosgrove SE. Association of adverse events with antibiotic use in hospitalized patients. JAMA Intern Med 2017:177:1308-15.

12 Sikkens ], Van Agtmael M, Peters E et al. Assessment of appropriate antimicrobial prescribing: do experts agree? J Antimicrob Chemother 2016;71:2980-7.

13 Spivak ES, Cosgrove SE, Srinivasan A. Measuring appropriate antimicrobial use: attempts at opening the black box. Clin Infect Dis 2016;63:1639-44.

14 NHS England. Commissioning for Quality and Innovation (CQUIN) 2017/19. www.england.nhs.uk/nhs-standard-contract/cquin/cquin17-19/ [Accessed 26 October 2017].

15 Public Health England. Antimicrobial stewardship: Start smart then focus. PHE, 2011.

16 Powell N, Franklin BD, Jacklin A, Wilcock M. Omitted doses as an unintended consequence of a hospital restricted antibacterial system: a retrospective observational study. J Antimicrob Chemother 2015;70:3379-83.
17 Lim WS, Smith DL, Wise MP, Welham SA. British Thoracic Society community acquired pneumonia guideline and the NICE pneumonia guideline: how they fit together. BMJ Open Resp Res 2015;2:e000091.

18 Scottish Intercollegiate Guidelines Network. Sign 88: Management of suspected bacterial urinary tract infection in adults. SIGN, 2012 www.sign.ac.uk/assets/sign88.pdf [Accessed 9 August 2017].

19 Miyashita N, Shimizu H, Ouchi K et al. Assessment of the usefulness of sputum Gram stain and culture for diagnosis of communityacquired pneumonia requiring hospitalization. Med Sci Monit 2008:14:CR171-6

20 García-Vázquez E, Marcos MA, Mensa ] et al. Assessment of the usefulness of sputum culture for diagnosis of community-acquired pneumonia using the PORT predictive scoring system. Arch Intern Med 2004;164:1807-11.

21 Surviving Sepsis Campaign. Guidelines. www.survivingsepsis.org/ Guidelines/Pages/default.aspx [Accessed 9 August 2017].

22 Broom J, Broom A, Adams K, Plage S. What prevents the intravenous to oral antibiotic switch? A qualitative study of hospital doctors' accounts of what influences their clinical practice. J Antimicrob Chemother 2016;71:2295-9.

23 Schuts EC, Hulscher MEJL, Mouton JW et al. Current evidence on hospital antimicrobial stewardship objectives: a systematic review and meta-analysis. Lancet Infect Dis 2016;16:847-56.

24 Caterino JM, Ting SA, Sisbarro SG et al. Age, nursing home residence, and presentation of urinary tract infection in U.S. emergency departments, 2001-2008. Acad Emerg Med 2012;19:1173-80.

25 Fridkin S, Baggs ], Fagan R et al. Vital signs: improving antibiotic use among hospitalized patients. MMWR 2014;63:194-200.

$26 \operatorname{Lim} \mathrm{VH}$, Whitehurst T, Usoro E, Ng SM. Management of urinary tract infections in elderly patients: Strategies for improvement. BMJ Qual Improv Rep 2014;3:u203314.w1503.

27 Spellberg B. The New Antibiotic Mantra - 'Shorter Is Better;' JAMA Intern Med 2016;176:1254-5.

28 National Institute for Health and Care Excellence. Pneumonia in adults: diagnosis and management. NICE, 2014.

29 Kalil AC, Metensky ML, Klompas M et al. Management of adults with hospital-acquired and ventilator-associated pneumonia: 2016 Clinical Practice Guidelines by the Infectious Diseases Society of America and the American Thoracic Society. Clin Infect Dis 2016;63:1-51.

30 Global Initiative for Chronic Obstructive Lung Disease. Pocket Guide to COPD Diagnosis, Management and Prevention. A Guide for Healthcare Professionals. Global Initiative for Chronic Obstructive Lung Disease, Inc, 2017.

31 Murphy D, Overton E, Steinberg J, Jacob J. Effect of blood culture acquisition time relative to antibiotic administration on diagnostic yield. Crit Care Med 2015;43:1-45.

32 Dryden M, Saeed K, Townsend R et al. Antibiotic stewardship and early discharge from hospital: impact of a structured approach to antimicrobial management. J Antimicrob Chemother 2012;67:2289-96

33 National Institute of Health and Care Excellence. Urinary tract infections in adults. Quality standard [QS90]. NICE, 2015.

Address for correspondence: Mr Neil Powell, Pharmacy Department, Royal Cornwall Hospital, Truro TR1 3LJ, UK. Email: neil.powell2@nhs.net 